Bio - grafia. Escritos sobre la Biología y su Enseñanza. ISSN 2027

Edición Extraordinaria. p.p. 771 - 784

Memorias del VIII Encuentro Nacional de Experiencias en Enseñanza de la Biología y la Educación Ambiental. III Congreso Nacional de Investigación en Enseñanza de la Biología.

\title{
ACTITUDES PRO AMBIENTALES EN LOS NIÑOS Y NIÑAS DE SEXTO GRADO. UN APORTE A LA EDUCACIÓN AMBIENTAL EN EL COLEGIO KIMY PERNIA DOMICÓ IED - JM
}

\author{
PRO-ENVIRONMENTAL ATTITUDES IN CHILDREN; A CONTRIBUTION TO \\ ENVIRONMENTAL EDUCATION AT SCHOOL KIMY PERNIA DOMICÓ JM
}

Barreto Tovar Carlos Humberto y García Donato Andrea del Pilar ${ }^{2}$.

RESUMEN

0

P

0

$N$

E

$N$

c

I

A

Este escrito recoge los resultados de la investigación desarrollada en el año 2014 con los estudiantes del curso 603 del IED Kimy Pernía Domicó Jornada Mañana, con el propósito de reconocer y analizar sus actitudes proambientales. El abordaje desde el punto de vista metodológico, se encuadra en la Investigación Acción Educativa; esto con el desarrollo de una etapa inicial de diagnóstico y el desarrollo posterior de tres estrategias para el reconocimiento y análisis respectivo del componente cognoscitivo, afectivo y connativo de las actitudes proambientales de los niños y las niñas participantes del proceso. La evaluación de la implementación del programa de actividades resultó positiva, en tanto se reconocen y analizan las actitudes proambientales en los niños y niñas participantes como punto de partida para el fortalecimiento de sus actitudes y comportamientos frente al ambiente. A la par, las maestras involucradas en el proceso fortalecen sus propias actitudes proambientales y cambian su mirada sobre los estudiantes, el contexto y las propias dinámicas del aula.

PALABRAS CLAVE: Conocimientos ambientales, actitudes proambientales, comportamientos proambientales, educación ambiental, Proyecto Ambiental Escolar (PRAE).

${ }^{1}$ Universidad de la Sabana. Facultad de Educación. Especialización en Pedagogía e Investigación en el Aula. Docente - Investigador Correo: carloshumbertobarreto@hotmail.com

2 Universidad de la Sabana. Facultad de Educación. Especialización en Pedagogía e Investigación en el Aula. Coinvestigadora. Docente Secretaria de Educación de Bogotá. Docente - investigadora Corporación Universitaria Minuto de Dios - Uniminuto. Correo: andreita.gardo@gmail.com 
Bio - grafia. Escritos sobre la Biología y su Enseñanza. ISSN 2027

Edición Extraordinaria. p.p. 771 - 784

Memorias del VIII Encuentro Nacional de Experiencias en Enseñanza de la Biología y la Educación Ambiental. III Congreso Nacional de Investigación en Enseñanza de la Biología.

\begin{abstract}
This paper presents the results of research carried in 2014 with out sixth grade morning students (603) from the school IED Kimy Pernía Domicó, in order to recognition and analysis their pro-environmental attitudes. The approach from the methodological point of view was made take into account the Educational Action Research; therefore an idea from a general improvement action plan was established; this is the development of an initial assessment phase or diagnosis and subsequent development of three strategies for the respective cognitive strengthening, affective and normative (connativo) component of pro-environmental attitudes of boys and girls participating in the process. The evaluation of the program's implementation of activities was positive; while they are recognizing and analyzing the pro-environmental attitudes among participating children as a starting point for strengthening their attitudes and behavior towards the environment. At the same time, the teachers involved in the process strengthen their own pro-environmental attitudes and change their look on the students, the context and own classroom dynamics.
\end{abstract}

KEY WORDS: Environmental knowledge, eco-friendly attitude, pro-environmental behavior, environmental education, School Environmental Project

\title{
INTRODUCCIÓN
}

Este artículo se considera un aporte a los procesos de educación ambiental que se desarrollan dentro de la institución en el marco del Proyecto Ambiental Escolar (PRAE) "En búsqueda de sentido de pertenencia, hacia la construcción de una cultura ambiental en la comunidad educativa de la Institución Kimy Pernía Domicó". Para la investigación se partió de reconocer el contexto a intervenir, dadas las problemáticas ambientales que caracterizan el sector donde se encuentra el colegio por la cría de ganado porcino y bovino, la quema de cables para la extracción del cobre y la disposición inadecuada de residuos en la ronda del río. Considerando esto, se formula un problema de investigación, centrado en la falta de apropiación por los estudiantes de los discursos de cuidado y conservación del medio ambiente; evidenciada en sus acciones frente a la disposición de los residuos sólidos y el uso racional del recurso hídrico. 
Bio - grafia. Escritos sobre la Biología y su Enseñanza. ISSN 2027

Edición Extraordinaria. p.p. $771-784$

Memorias del VIII Encuentro Nacional de Experiencias en Enseñanza de la Biología y la Educación Ambiental. III Congreso Nacional de Investigación en Enseñanza de la Biología.

Se fundamentó en el modelo de la Teoría de la Acción Razonada (TAR) que involucra factores como las creencias, las actitudes, las normas, la motivación y la intención. Por lo cual se pretendió a través de las estrategias implementadas (diagnóstico y reconocimiento y análisis de los componentes de las actitudes proambientales de los participantes del proceso) vincular esos factores para el cumplimiento del propósito de la investigación, bajo la premisa de que al reconocer y analizar las actitudes se podrían aplicar actividades para fortalecerlas y con esto estimular comportamientos proambientales, ya que la TAR permite analizar los procesos que llevan de una actitud a una conducta, buscando el origen de la conducta en las creencias que el individuo mantiene ante la intención de realizarla. En ese orden de ideas, se vincularon tres categorías de partida a saber; conocimientos ambientales, actitudes ambientales y comportamientos proambientales.

Los conocimientos ambientales, en gran medida determinan las creencias, actitudes y comportamientos que la persona manifiesta frente al medio ambiente. Ellos se constituyen con base en las experiencias de vida de cada individuo y su relación con los otros, en sus espacios familiares, escolares y laborales. Para Febles (1999) Citada por GARCÍA (2006) "El conocimiento ambiental es un proceso complejo, que incluye la obtención, análisis y sistematización por parte del individuo de la información proveniente de su entorno, social por naturaleza, este constituye un paso importante para su comprensión a través de acciones concretas, que a su vez, influyen en el desarrollo de estos conocimientos". De esta forma se puede afirmar que los procesos de educación ambiental, permiten que esa información ambiental recibida por los individuos, se materialice en conocimientos que contribuyen a la consolidación de actitudes proambientales.

Dichas actitudes proambientales, son entendidas desde Zimmerman (2005) "...como una disposición relativamente durable y estable en el individuo frente a un objeto social ambiental determinado. El concepto de actitud apunta esencialmente a un proceso psico - socio - ambiental de evaluación del individuo frente al ambiente externo, con fines adaptativos y para las tomas de decisión en sus locomociones diarias". Dicho proceso evaluativo se estructura alrededor de tres componentes; uno cognoscitivo que vincula elementos informativos y experiencias que permiten que el individuo pueda pensar en términos de opiniones, juicios y creencias frente a la calidad de vida del entorno; un componente afectivo que involucra los sentimientos y emociones que suscitan en el individuo los entornos dependiendo de sus atributos; y un último componente, el connativo que se refiere a las disposiciones a actuar frente a un ambiente físico o socio cultural; en forma positiva o negativa a lo socialmente indicado. Estos tres componentes integrados armónicamente llevan a una actitud fuertemente estructurada. 
Bio - grafia. Escritos sobre la Biología y su Enseñanza. ISSN 2027

Edición Extraordinaria. p.p. 771 - 784

Memorias del VIII Encuentro Nacional de Experiencias en Enseñanza de la Biología y la Educación Ambiental. III Congreso Nacional de Investigación en Enseñanza de la Biología.

Finalmente, los comportamientos proambientales son definidos como un "conjunto de acciones intencionales, dirigidas y efectivas que responden a requerimientos sociales $e$ individuales que resultan de la protección del medio" (Corral - Verdugo, 2000) Citado por MARTINEZ J (2004). Estos comportamientos se dan ante exigencias del entorno social de los individuos, es decir, se rigen por las normas sociales y se determinan por: actitudes proambientales, emociones a favor de la naturaleza y su conservación, afinidad hacia lo natural, aprecio por la diversidad, motivos pro ecológicos, intenciones a actuar de manera pro ambiental y creencias ambientales.

El proceso de investigación pretendió el reconocimiento y análisis de las actitudes proambientales de los niños y las niñas del curso 603 de IED Kimy Pernía Domicó, en aras de su fortalecimiento, dado que el colegio se encuentra ubicado en un sector que presenta una grave problemática ambiental y se observa en los estudiantes un conocimiento en el tema pero unas acciones poco coherentes con ese conocimiento, en términos de la disposición inadecuada de los residuos y el uso inadecuado del residuo hídrico.

\section{METODOLOGÍA}

El trabajo se desarrolló en la Institución Educativa Distrital Kimy Pernía Domicó, ubicado en el costado sur del Barrio Potreritos, Unidad de Planeación Zonal Tintal Sur de la localidad de Bosa, al sur de Bogotá DC. Cuenta con capacidad para 3600 niños, niñas y adolescentes, desde grado cero hasta undécimo en jornadas mañana y tarde. El nombre del colegio es un homenaje a Kimy Pernía Domicó líder de los indígenas Embera Katío del alto Sinú, en la lucha contra la construcción de la represa hidroeléctrica de Urrá I, en el departamento de Córdoba (Colombia) asesinado por paramilitares en el 2001 en Tierralta. El colegio cuenta con muy buena infraestructura y dotación: 36 aulas académicas, 3 aulas de tecnología, 3 aulas de sistemas, 2 laboratorios de ciencias, 2 laboratorios de física y química, 1 aula de artes, 1 aula de danzas, 1 cancha de futbol en césped, 2 canchas múltiples en cemento, 1 biblioteca con estanterías al alcance de los estudiantes, 1 sala de lectura infantil ubicada en el segundo piso de la biblioteca, 1 sala doble de 
Bio - grafia. Escritos sobre la Biología y su Enseñanza. ISSN 2027

Edición Extraordinaria. p.p. $771-784$

Memorias del VIII Encuentro Nacional de Experiencias en Enseñanza de la Biología y la Educación Ambiental. III Congreso Nacional de Investigación en Enseñanza de la Biología.

audiovisuales con capacidad para 250 personas sentadas, comedor escolar con capacidad para atender 300 personas en simultáneo, granja escolar y zonas verdes.

El grupo con el que se adelantó la investigación, es el curso 603 de la jornada mañana. Un grupo homogéneo en la distribución por sexo, igualmente en términos de la edad solo dos niños se encuentran por fuera del rango de edad para el grado que cursan. Como particular se tiene la presencia de tres estudiantes en condición de desplazamiento y otros dos estudiantes de origen afro descendiente. Asimismo, es de resaltar que 12 estudiantes, es decir, cerca del 35\% acompañan a sus padres en el trabajo. También el $35 \%$ inician el año escolar con compromiso ya sea académico o convivencial, información que se obtiene del ejercicio de caracterización que se hace al inicio de cada año escolar.

Considerando este panorama, donde las problemáticas ambientales exigen a la comunidad educativa la implementación de estrategias de intervención, se genera la propuesta de investigación cuyos resultados se presentan en este escrito.

En aras de construir la propuesta metodológica se da una mirada a la investigación educativa, como práctica que a través de sus diferentes enfoques y perspectivas, ha aportado a la producción de conocimiento entorno a la escuela en todas sus dimensiones. Sin embargo, este tipo de investigación en algún momento empezó a ser encontrada por los profesores como poco significativa y muy limitada a la descripción, por la forma en que se venía desarrollando. Tampoco encontraban en ella soluciones a las problemáticas propias del aula, ya que se percibía como un proceso complicado que era adelantado por otros, a los que se consideraba expertos en el tema. En ese contexto, surge la investigación acción educativa como alternativa, en palabras de PARRA, C (2002) "El diseño metodológico de la investigación - acción - educativa se centra en la reflexión sistemática sobre la acción", lo cual solucionaba la inquietud frente a los tiempos para desarrollarse, ya que esta la harían los propios profesores en su aula, sobre los temas de su interés y les ayudaría a solucionar los problemas prácticos y reales que evidenciaban en el día a día.

Con base en la ruta de la Investigación Acción Educativa (IAE), se determinó para el desarrollo de la investigación la idea de mejora, la cual se fundamentó en la definición de actitud de Zimmermann (2007) para quien una actitud es "... una disposición relativamente durable y estable en el individuo frente a un objeto social ambiental determinado. El concepto de actitud apunta esencialmente a un proceso psico - socio - ambiental de evaluación del individuo frente a un objeto social ambiental determinado". Dicho proceso evaluativo considera tres componentes: 
Bio - grafia. Escritos sobre la Biología y su Enseñanza. ISSN 2027

Edición Extraordinaria. p.p. 771 - 784

Memorias del VIII Encuentro Nacional de Experiencias en Enseñanza de la Biología y la Educación Ambiental. III Congreso Nacional de Investigación en Enseñanza de la Biología.

cognoscitivo, afectivo y connativo, a partir de los cuales se definieron siguientes estrategias y sus correspondientes actividades:

Estrategia $N^{\circ} 1$. Diagnóstico: Aplicación del Instrumento de medición de actitudes pro ambientales y análisis de los resultados de la aplicación del instrumento.

Se empleó la Escala de Medición de Actitudes Pro ambientales Infantiles - EMAPI. EI instrumento consta de 18 ítems evaluados por una escala tipo Likert de 4 puntos que identifica un grado creciente de actitud ambiental (asignando 1 a la respuesta que refleja menor actitud y 4 a la que refleja mayor actitud) por lo que sus resultados pueden variar de una suma mínima de 18 puntos hasta una suma máxima de 72 puntos; el contenido de los ítems contempla elementos afectivos, cognitivos y conativos de la formación de la actitud/conducta, en relación con cinco temas ambientales: conservación, reciclaje, derechos/protección de los animales, apreciación de la naturaleza y contaminación, según Campos, M., Pasquali, C., Peinado, S. (2008). La finalidad de esta estrategia era reconocer y analizar las actitudes proambientales de los participantes a fin de establecer el programa de actividades a implementar con el fin de fortalecerlas.

Estrategia $N^{\circ} 2$. Componente cognoscitivo de las actitudes proambientales de los niños $y$ niñas

Taller Conservación del recurso hídrico y Taller Manejo de residuos sólidos. Teniendo en cuenta que el manejo del recurso hídrico y la disposición de residuos sólidos fueron los temas que en los diarios de campo se encontraron como críticos, se determinó trabajar el componente cognitivo a partir de ellos. Para esto se diseñó un taller de conservación del recurso hídrico tomando elementos de talleres consultados y se concertó con el referente ambiental del Hospital Pablo VI de Bosa la realización de dos sesiones de taller sobre manejo integral de residuos sólidos en el marco de la implementación del programa Basura Cero.

Estrategia $\mathrm{N}^{\circ} 3$. Componente afectivo de las actitudes proambientales de los niños y niñas.

Taller Cartografía social y Taller de Siembra. Se escogieron estas actividades para trabajar lo afectivo, pensando en el reconocimiento y apropiación del territorio, dadas las potencialidades del mismo, la cercanía de los niños con el tema de la agricultura y el hecho de que el colegio cuente con granja.

Estrategia $\mathrm{N}^{\circ} 4$. Componente connativo de las actitudes proambientales de los niños y niñas 
Bio - grafia. Escritos sobre la Biología y su Enseñanza. ISSN 2027

Edición Extraordinaria. p.p. 771 - 784

Memorias del VIII Encuentro Nacional de Experiencias en Enseñanza de la Biología y la Educación Ambiental. III Congreso Nacional de Investigación en Enseñanza de la Biología.

Decálogo de los comportamientos proambientales del IED Kimy Pernía Domicó. En la consideración que fortaleciendo la actitud se fortalecía el comportamiento proambiental y contando con los conocimientos ambientales de los participantes se les pide formular el listado de acciones que constituyen los comportamientos proambientales deseables para el IED Kimy Pernía Domicó.

\section{RESULTADOS Y DISCUSIÓN}

\section{Estrategia $N^{\circ} 1$. Diagnóstico: Aplicación del Instrumento de medición de actitudes pro ambientales y análisis de los resultados de la aplicación del instrumento.}

La implementación de la primera estrategia buscaba obtener un diagnóstico que sirviera como punto de partida para determinar la pertinencia de las actividades programadas. Por lo tanto se aplicó la Escala de Medición de Actitudes Pro ambientales Infantiles - Emapi, Campos, M., Pasquali, C., Peinado, S. (2008) que se describió en la metodología.

En términos generales, se pudo apreciar que los ítems que resultaron en actitud pro ambiental negativa son aquellos que exigen de las personas mayor compromiso en las acciones; fabricar viviendas para los pájaros, recoger la basura de los patios y compartir el carro. Los demás temas se inclinaron hacia la actitud pro ambiental positiva, por lo tanto un total del $90 \%$ de los estudiantes que aplicaron el instrumento tienen actitudes pro ambientales positivas que pueden fortalecerse mediante el proceso propuesto. Para Cheng y Monroe (2012) citados por Gallí (2013) en estos análisis se debe considerar "la importancia de experiencias pasadas en la naturaleza, la proximidad del lugar de residencia a la naturaleza, el conocimiento ambiental y la influencia de los valores parentales respecto a la naturaleza como predictores de la intención de participar de actividades ambientalmente responsables, o sea, de comportamientos proambientales en la niñez", como se mencionó algunas familias de los participantes tienen sus raíces en el campo y el contexto del colegio conserva fuertes elementos de ruralidad como lo es la ronda del río y el humedal. 
Bio - grafia. Escritos sobre la Biología y su Enseñanza. ISSN 2027

Edición Extraordinaria. p.p. 771 - 784

Memorias del VIII Encuentro Nacional de Experiencias en Enseñanza de la Biología y la Educación Ambiental. III Congreso Nacional de Investigación en Enseñanza de la Biología.

Estrategia $N^{\circ} 2$. Componente cognoscitivo de las actitudes proambientales de los niños y niñas: Taller Conservación del recurso hídrico y Taller Manejo de residuos sólidos.

La implementación de la estrategia N² tenía como propósito el componente cognitivo de la actitud. Por lo tanto, se desarrollaron dos talleres en tres sesiones para abordar el tema de recurso hídrico y residuos sólidos. Se encontró que en el 41,7 \% de las 139 intervenciones analizadas los estudiantes ponen de manifiesto alguna información o conocimiento sobre la situación abordada es decir, intervienen desde el componente cognitivo "Porque sin agua todas las plantas se secarían por falta de agua y el oxígeno no sería respirable" Diario de campo, no obstante el $36,7 \%$ se expresa desde lo que considera está bien o mal pero se limita a ello sin argumentar más profundamente, es decir, desde lo afectivo "Debemos cuidar el agua porque se está agotando y no tendríamos agua" Diario de campo. El 21, 6 se refiere a las situaciones en términos de lo que se debe o se tiene que hacer, ósea desde el componente connativo, "No debemos jugar con el agua" Diario de campo.

En cuanto a los temas ambientales, el $58,3 \%$ de las intervenciones se enfocaron en el tema conservación, ya que se mencionaba el agua en términos de la necesidad de su cuidado o de su uso sin profundizar en causas o consecuencias "Deberíamos cuidar el agua o se acabaría el agua para siempre y moriríamos de sed" Diario de campo. Galli F. y otros, (2013) citan a Corral Verdugo (2012) quien "enfatiza que este tipo de comportamiento encaja en lo que se refiere a comportamientos frugales o austeros, es decir, se reduce el consumo de recursos de manera voluntaria con el objetivo de que ocurra menos desperdicio y a la vez beneficie el medio ambiente. Por otro lado, solo el $2,2 \%$ se mencionó el agua en términos del reciclaje "Pues que no hay que desperdiciar el agua y aprender a reutilizarla y ojalá la gente lo entendiera" Diario de campo, el $7,2 \%$ la relacionaron son el derecho o la necesidad que tienen de ella otras especies animales "Estamos contaminando el agua y los afectados son los animales" Diario de campo, el 15\% desde la apreciación de la naturaleza en su conjunto, el planeta o el mundo "El agua se está acabando y la tierra se está secando" Diario de campo, y el $17 \%$ en relación a su posible contaminación "Que no echen basura a los ríos porque contaminan el medio ambiente" Diario de campo. 
Bio - grafia. Escritos sobre la Biología y su Enseñanza. ISSN 2027

Edición Extraordinaria. p.p. 771 - 784

Memorias del VIII Encuentro Nacional de Experiencias en Enseñanza de la Biología y la Educación Ambiental. III Congreso Nacional de Investigación en Enseñanza de la Biología.

Estos resultados permiten ver que existe en los estudiantes un conocimiento básico que les permite analizar las situaciones propuestas y que en la mayoría de los casos la postura se centra en la necesidad del agua como recurso. En ese sentido, se aprecia que la implementación de la segunda estrategia cumple con su objetivo ya que las actividades permitieron la ejercitación de conceptos propios del tema ambiental. Según Hart (1995) citado por Galli F. y Otros, (2013), "los niños pueden convertirse en importantes activistas ambientales, pues comprenden la necesidad de ofrecer menos riesgo al medio ambiente y no ven barreras para esto. Eso se explica por el hecho de que los niños son más abiertos al cambio y menos inmersos en el sistema económico y en el orden social establecido, hechos que pueden ser considerados como obstáculos de la sostenibilidad.

\section{Estrategia $\mathrm{N}^{\circ} 3$. Componente afectivo de las actitudes proambientales de los niños y niñas: Taller Cartografía social y Taller de Siembra.}

En la implementación de la estrategia $N^{0} 3$ que pretendía trabajar el componente afectivo de la actitud se desarrollaron dos actividades: primero, ejercicio de cartografía social y segundo un Taller de Siembra.

En la cartografía social, se proponen los componentes socio cultural, físico natural y económico. En el primer componente, se mencionan pocos espacios de encuentro socio cultural, de hecho esta actividad para los estudiantes está muy centralizada en el colegio. No obstante, esto se aprecia como valioso ya que "El contexto escolar, en cuanto espacio de aprendizaje, tiene un rol importante en la formación de futuros sujetos más involucrados con los temas medio ambientales, sea por el espacio con más presencia de naturaleza o por el programa educacional" Galli F. y Otros, (2013), y el colegio cumple con ser un espacio con fuerte presencia de elementos rurales y en coherencia con este contexto se desarrollan proyectos de formación en temas afines a lo ambiental como la granja y el manejo de material reciclable.

En el componente físico natural, los estudiantes mencionan: ronda del río, humedal, animales, potreros aledaños al rio, construcción junto al colegio, ciclo rutas, canchas del colegio, potreros cercanos a la nueva construcción, algunos señalan la ruta del carro de la basura. La presencia del río, su ronda y el humedal son ampliamente reconocidos por los estudiantes ya que estos determinan el hecho de que haya cría de ganado en sus alrededores, actividad agrícola y zonas 
Bio - grafia. Escritos sobre la Biología y su Enseñanza. ISSN 2027

Edición Extraordinaria. p.p. 771 - 784

Memorias del VIII Encuentro Nacional de Experiencias en Enseñanza de la Biología y la Educación Ambiental. III Congreso Nacional de Investigación en Enseñanza de la Biología.

despobladas que se usan para la quema de basuras y materiales para el reciclaje. Situaciones estas que han generado la problemática ambiental que caracteriza al sector. Finalmente, en el componente económico, los estudiantes destacan: cría de cerdos y vacas, cultivo de algunas hortalizas y algunos pocos negocios de mercado o papelería. Para Galli F. y Otros, (2013), "Los niños que tienen más contacto con la naturaleza presentan una preocupación más real y concreta acerca de los daños hacia la naturaleza", por lo tanto se observa en los resultados que la estrategia implementada sirvió para volver la mirada sobre el contexto y que este incida en las actitudes proambientales de los participantes, a través de su reconocimiento y apropiación.

Igualmente, en el marco de esta estrategia se realizó la actividad de la siembra, la cual permitió consolidar el proceso en el sentido en que se contó con los conceptos que los estudiantes ejercitaron a lo largo del proceso y con el reconocimiento del territorio dado en la cartografía social. Para García y Priotto (2009) "... podemos considerar que la forma de pensar y saber sobre el ambiente es la resultante de un conjunto de significaciones, normas, valores, intereses y acciones socioculturales. La percepción sobre el ambiente se construye en el seno de una sociedad y se concreta y modifica en una interrelación permanente entre los procesos simbólicos y las prácticas cotidianas".

Los participantes se conectan con la actividad y aportan desde sus conocimientos y experiencias de vida, poniendo de manifiesto esas significaciones, normas, valores, intereses y acciones que determinan su percepción sobre el ambiente, fortaleciendo su vínculo emocional con el mismo.

Estrategia $\mathrm{N}^{\circ} 4$. Componente connativo de las actitudes proambientales de los niños y niñas: Decálogo de los comportamientos proambientales del IED Kimy Pernía Domicó.

La estrategia $\mathrm{N}^{\circ} 4$ tuvo como objetivo el componente connativo de las actitudes proambientales de los estudiantes participantes. Para ello, tras una reflexión sobre el proceso adelantado, se pide a los estudiantes mencionar las acciones que consideran deberían constituir el decálogo de los Comportamientos proambientales del IED Kimy Pernía Domicó. Se entiende por comportamientos proambientales desde Corral - Verdugo, (2000) Citado por Martínez (2004), un "conjunto de acciones intencionales, dirigidas y efectivas que responden a requerimientos sociales $e$ individuales que resultan de la protección del medio", en este caso serían comportamientos deseables en los estudiantes del colegio, es decir, serían acciones intencionales dirigidas a actuar positivamente con relación al ambiente. 
Bio - grafia. Escritos sobre la Biología y su Enseñanza. ISSN 2027

Edición Extraordinaria. p.p. 771 - 784

Memorias del VIII Encuentro Nacional de Experiencias en Enseñanza de la Biología y la Educación Ambiental. III Congreso Nacional de Investigación en Enseñanza de la Biología.

Para el análisis se tomaron los 37 enunciados formulados por los estudiantes y se contrastaron con los 16 tipos de conductas pro ecológicas que describe Corral, V (2010): Reducción en el consumo de productos, Reúso de desechos, Reciclaje, Acciones de estética ambiental, Compra de productos amigables para el ambiente, Elaboración de composta, Ahorro de agua, Ahorro de energía eléctrica, Disminución del uso de automóviles, Ahorro de combustible, Lectura de tópicos ambientales, Persuasión pro ecológica, Cabildeo pro ambiental, Diseño y construcción pro ecológicos, Cuidado de ecosistemas y Planificación familiar.

Así, se encontró que los 37 enunciados formulados por los estudiantes se relacionan con 5 de esas 16 conductas proambientales de la siguiente forma; 2 enunciados referidos a la reducción en el consumo de productos "No usar tanto químico en tu casa" Diario de campo, 2 al reúso de desechos "Recoger agua cuando llueve para lavar la ropa" Diario de campo, 6 al ahorro de agua "Ahorrar el agua porque se acaba" Diario de campo, 22 a la persuasión pro ecológica "Cuidar el agua porque gota a gota se agota" Diario de campo y 5 al cuidado de los ecosistemas "Cuidando la naturaleza vivimos mejor" Diario de campo.

Para Corral, V (2010) la conducta pro ecológica se da ante exigencias del entorno social de los individuos, es decir, se rige por las normas sociales y estas conductas están determinadas por: actitudes proambientales, emociones a favor de la naturaleza y su conservación, afinidad hacia lo natural, aprecio por la diversidad, motivos pro ecológicos, intenciones a actuar de manera pro ambiental, creencias ambientales y las normas sociales.

En ese orden de ideas, los comportamientos proambientales propuestos por los estudiantes serían la expresión de sus actitudes, emociones, afinidades, motivos, intenciones, creencias y normas, las cuales estarían mayoritariamente centradas en el ahorro de agua y en la persuasión pro ecológica. Este resultado se asume como satisfactorio, ya que si bien estos comportamientos o conductas no se trabajaron en forma explícita, los estudiantes a través del proceso llegaron a ellos. 
Bio - grafia. Escritos sobre la Biología y su Enseñanza. ISSN 2027

Edición Extraordinaria. p.p. 771 - 784

Memorias del VIII Encuentro Nacional de Experiencias en Enseñanza de la Biología y la Educación Ambiental. III Congreso Nacional de Investigación en Enseñanza de la Biología.

\section{CONCLUSIONES}

El proceso desarrollado permite llegar a las siguientes conclusiones:

Se reconocieron y analizaron las actitudes proambientales existentes en los participantes, a partir de la implementación de cuatro estrategias a saber: una estrategia inicial centrada en obtener un diagnóstico de las actitudes proambientales de los estudiantes participantes, y tres estrategias restantes cada una enfocada a cada uno de los componentes de la actitud: el cognoscitivo, el afectivo y el connativo, según lo expuesto en el marco teórico.

El diseño e implementación de las estrategias, vinculó el desarrollo de una serie de actividades enfocadas al reconocimiento y apropiación del territorio, es decir, la investigación no se limitó al manejo de unos conceptos técnicos sobre el tema medioambiental, sino que por el contrario los procesos reflexivos se dieron alrededor de la propia realidad de los estudiantes, permitiéndoles reconocer el territorio que habitan con sus problemáticas y potencialidades, entendiendo que en esas dinámicas él mismo incide desde cada uno de los momentos de su vida cotidiana. Reconociendo de esta forma los componentes cognitivo, afectivo y connativo de las actitudes proambientales de los participantes. 
Edición Extraordinaria. p.p. 771 - 784

Memorias del VIII Encuentro Nacional de Experiencias en Enseñanza de la Biología y la Educación Ambiental. III Congreso Nacional de Investigación en Enseñanza de la Biología.

La evaluación de la implementación del programa de actividades arroja un resultado positivo, en tanto se reconocen y analizan las actitudes proambientales de los niños y niñas participantes, lo cual puede constituir un punto de partida para que en la práctica sus actitudes y comportamientos frente al ambiente se fortalezcan. Asimismo las maestras involucradas en el proceso fortalecen sus propias actitudes proambientales y cambian su mirada sobre los estudiantes, el contexto y las propias dinámicas del aula; se reconoce el estudiante como un sujeto poseedor de un conocimiento ambiental constituido en sus experiencias de vida y su interacción con el territorio, se mira el contexto desde sus problemáticas pero reconociendo igual sus potencialidades y se cuestionan las dinámicas del aula para pensar en apuestas más horizontales que llamen a los estudiantes a participar más activamente en la gestación de su propio aprendizaje. 
Bio - grafia. Escritos sobre la Biología y su Enseñanza. ISSN 2027

Edición Extraordinaria. p.p. 771 - 784

Memorias del VIII Encuentro Nacional de Experiencias en Enseñanza de la Biología y la Educación Ambiental. III Congreso Nacional de Investigación en Enseñanza de la Biología.

\section{REFERENCIAS}

CAMPOS, M., PASQUALI, C., PEINADO, S. (2008). Evaluación Psicométrica de un Instrumento de Medición de Actitudes Pro Ambientales en Escolares Venezolanos. Universidad Simón Bolivar: Caracas, Venezuela. Recuperado el 22 de abril de:

http://www.scielo.org.ve/pdf/pdg/v29n2/art08.pdf

CORRAL, V. (2010). Psicología de la sustentabilidad. Un análisis de lo que nos hace proecológicos y pro sociales. Trillas. México.

GARCÍA A. (2014). Diario de campo de la investigación. Documento Inédito.

GARCÍA D y PRIOTTO G, (2009). Educación Ambiental: Aportes políticos y pedagógicos en la construcción del campo de la Educación Ambiental. Jefatura de Gabinete de Ministros, Secretaría de Ambiente y Desarrollo Sustentable. Buenos Aires, Argentina.

GALLI F, y Otros, (2013). Actitudes hacia el medio ambiente en la infancia: un análisis de niños del sur de Brasil. Universidad Federal do Rio Grande Do Sul, Brasil MARTÍNEZ, J (2004). Comportamiento proambiental. Una aproximación al estudio del desarrollo sustentable con énfasis en el comportamiento. Persona - ambiente (1). Theomai, invierno.

PARRA, C (2002). Investigación Acción y desarrollo profesional. Educación y Educadores volumen $N^{\circ} 5$. Universidad de la Sabana.

ZIMMERMAN, M (2005). Ecopedagogía: el planeta en emergencia. Ecoediciones. Bogotá, Colombia 
